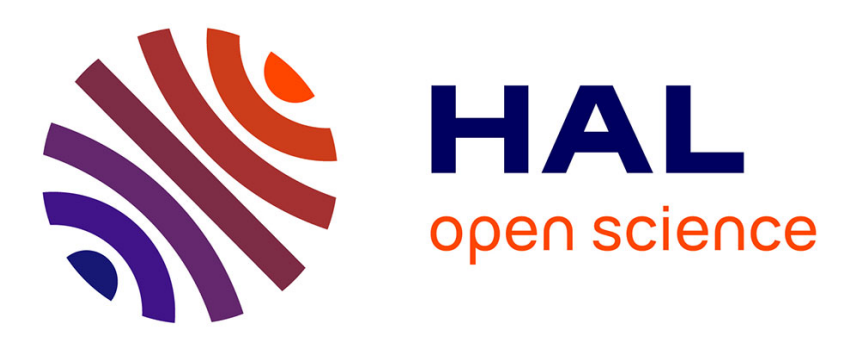

\title{
Change of concavity of the field temperature transition lines at short times in the Eu0.4Sr0.6S spin glass
}

\author{
J. Rajchenbach, N. Bontemps
}

\section{To cite this version:}

J. Rajchenbach, N. Bontemps. Change of concavity of the field temperature transition lines at short times in the Eu0.4Sr0.6S spin glass. Journal de Physique Lettres, 1983, 44 (18), pp.799-804. 10.1051/jphyslet:019830044018079900 . jpa-00232265

\section{HAL Id: jpa-00232265 https://hal.science/jpa-00232265}

Submitted on 1 Jan 1983

HAL is a multi-disciplinary open access archive for the deposit and dissemination of scientific research documents, whether they are published or not. The documents may come from teaching and research institutions in France or abroad, or from public or private research centers.
L'archive ouverte pluridisciplinaire HAL, est destinée au dépôt et à la diffusion de documents scientifiques de niveau recherche, publiés ou non, émanant des établissements d'enseignement et de recherche français ou étrangers, des laboratoires publics ou privés. 
Classification

Physics Abstracts

$75.40-64.60-78.20 \mathrm{~L}$

\title{
Change of concavity of the field temperature transition lines at short times in the $\mathrm{Eu}_{0.4} \mathrm{Sr}_{0.6} \mathrm{~S}$ spin glass
}

\author{
J. Rajchenbach and N. Bontemps \\ Laboratoire d'Optique Physique, ESPCI, 10, rue Vauquelin, 75231 Paris Cedex 05, France
}

(Reçu le 28 juin 1983, accepté le 20 juillet 1983)

\begin{abstract}
Résumé. - Nous avons mesuré la susceptibilité longitudinale et transverse dans $\mathrm{Eu}_{0,4} \mathrm{Sr}_{0,6} \mathrm{~S}\left(10^{2}\right.$ $10^{5} \mathrm{~Hz}$ ) sous champ appliqué. L'apparition d'une composante imaginaire permet de relever des lignes de transition champ/température, identiques dans les deux configurations; aux champs faibles, la concavité s'inverse et ce d'autant plus que la fréquence est plus élevée. L'interaction dipôle-dipôle permet d'expliquer l'isotropie de la réponse. Nous discutons les résultats en nous référant à la théorie de champ moyen et aux simulations numériques.
\end{abstract}

\begin{abstract}
We report on the transverse and longitudinal response in $\mathrm{Eu}_{0.4} \mathrm{Sr}_{0.6} \mathrm{~S}$ with a small a.c. field $\left(10^{2}-10^{5} \mathrm{~Hz}\right)$ superimposed to a large static one. From the onset of the imaginary susceptibility, we derive transition lines, identical in both configurations, which exhibit, at low field, a reversed curvature more pronounced with increasing frequency. The isotropy of the response is assigned to the dipolar interaction. Our results are compared to mean field theory and numerical simulations.
\end{abstract}

There is presently a fairly large amount of experimental work showing the existence of « De Almeida-Thouless » (AT) lines [1] in different spin-glass systems [2-7] using various experimental determinations. For the measurements which define the transition temperature $T_{\mathrm{G}}(H)$ as the temperature at which irreversible properties occur, time dependent lines have been found [6-7]. However, the experimental time scale affected only the field scale and not the shape of the line [7].

We report here on such dynamical measurements in the insulating system $\mathrm{Eu}_{0.4} \mathrm{Sr}_{0.6} \mathrm{~S}$ which shows clear evidence of a change of the shape of the line at low fields when the time scale is reduced from $10^{-3} \mathrm{~s}$ to $10^{-6} \mathrm{~s}$. Moreover, in an attempt to look for a possible transverse freezing as predicted by the meanfield theory $[8,9]$, we have also probed the transverse response of the system in presence of the magnetic field; we show that both the longitudinal and the transverse out of phase responses are the same. This must be due to the dipole-dipole interaction which strongly couples all degrees of freedom [10]. Our transverse measurements should thus be rather considered as a test of the anisotropy and not of the local response of the spins.

If we do not mention other kinds of arguments (such as space dimensionality for short range spin-glasses [11, 12] or the large dependence of the freezing temperature with frequency) it is because the similarity of the predictions of mean field theory $[8,9]$ and of numerical simulations $[13,14]$ is such that our experiments are not able to discriminate between them. 
We have measured simultaneously the real and imaginary component of the a.c. susceptibility ( $\chi^{\prime}$ and $\chi^{\prime \prime}$ respectively) when a small field $\Delta H$ modulated at the frequency $\omega$ is superimposed to a large static field $H$.

The sample is a single cubic crystal platelet $\left(3 \times 3 \times 0.7 \mathrm{~mm}^{3} ; 24.2 \mathrm{mg}\right) . H$ is always applied parallel to a $\langle 001>$ axis. We have probed two configurations : the longitudinal one $(\Delta H / / H)$ and the transverse one $(\Delta H \perp H)$. In both cases the magnetization $\Delta M$ associated to $\Delta H$ is measured by means of Faraday rotation [15]. This technique is a convenient one because of its quick response and great versatility concerning the relative orientation of $H$ and $\Delta H$. However it requires $\Delta H$ to be applied parallel to the light beam, hence perpendicular to the platelet ; therefore $H$ is either parallel or perpendicular to the platelet.

In order to make available the comparison between the two configurations we have measured on our sample the static magnetization in both configurations. We have thus derived empirical demagnetizing factors which allow us to plot real fields in place of applied fields. By taking this correction into account, $\Delta H \sim 0.2 \mathrm{Oe}$ and $H$ is varied from 1 to $50 \mathrm{Oe}$. However, as the correction on $H$ is quite dramatic since the demagnetizing field may reach $90 \%$ of the applied field, the absolute value of $H$ is not accurately defined : we estimate an uncertainty of $20 \%$ on the absolute value of the field $H$.

$\chi^{\prime}$ and $\chi^{\prime \prime}$ responses were recorded through a two-phase lock-in amplifier with a good phase stability $(0.02 \mathrm{dg})$, ensuring that $\chi^{\prime \prime}$ is not contaminated by $\chi^{\prime}$ though much smaller $\left(\chi^{\prime \prime} \sim 10^{-2} \chi^{\prime}\right)$. The sample was field cooled from $2.2 \mathrm{~K}$ down to $1.3 \mathrm{~K}$. Typical $\chi^{\prime \prime}$ curves are shown on figure 1 for 4 field values. As the onset of $\chi^{\prime \prime}$, when the temperature is decreased, is rather smooth, the corresponding temperature is not accurately defined. We thus chose a threshold value $L$ which, though small, is still sufficiently large so that any experimental phase artefact remains reasonably negligible compared to $L$. The transition temperature $T_{\mathrm{G}}(H, \omega)$ is then defined by $\chi^{\prime \prime}(H, T, \omega)=L$ (see Fig. 1).

It should be pointed out that in previous works $T_{\mathrm{G}}(H)$ has been defined either by the onset of $\chi^{\prime \prime}[6]$ or by the inflection point [16]. On our curves this latter point is rather badly defined. Moreover, we do not think it to exhibit any specific feature. We rather show on figure 2 a set of lines derived from our $\chi^{\prime \prime}$ data for two different threshold values (identified as $L_{1}$ and $L_{2}$ on Fig. 1). $L_{2}\left(>L_{1}\right)$ has been chosen for $\chi^{\prime \prime}(0, \omega)$ in order to lead to the zero field transition temperature

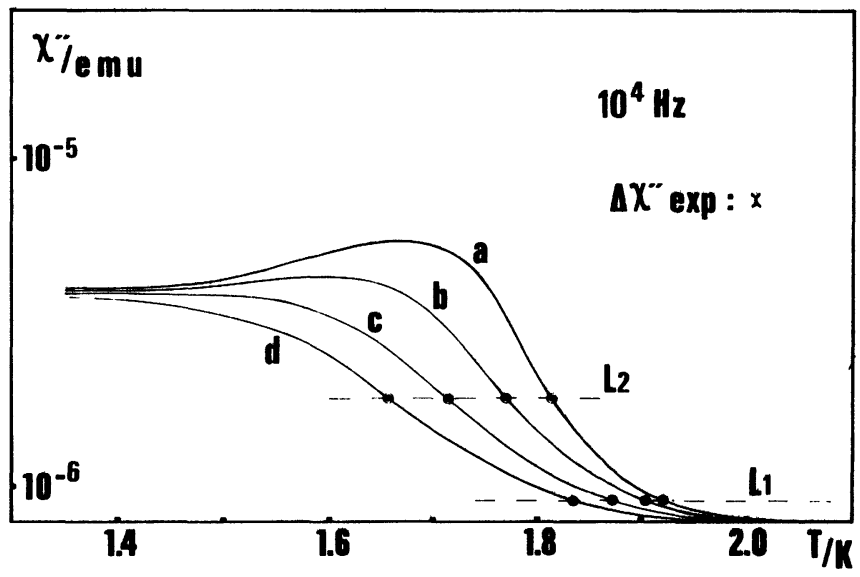

Fig. 1. $-\chi^{\prime \prime}(H, T)$ curves recorded as a function of temperature for the following static field values : $\left.a\right)$ $H=0 ; b) H=4 \mathrm{Oe} ; c) H=8 \mathrm{Oe} ; d) H=12 \mathrm{Oe} ; L_{1}$ and $L_{2}$ are the threshold values which have been chosen in order that : $T_{\mathrm{G}}\left(H=0, \omega, L_{2}\right)=T_{\mathrm{G}}\left(H=0,10^{-1} \omega, L_{1}\right)$. The experimental errors $\Delta \chi_{\text {exp }}^{\prime \prime}$ include phase shift contamination by $\chi^{\prime}$ and noise. The attempt field $\Delta H=0.2 \mathrm{Oe}$. 


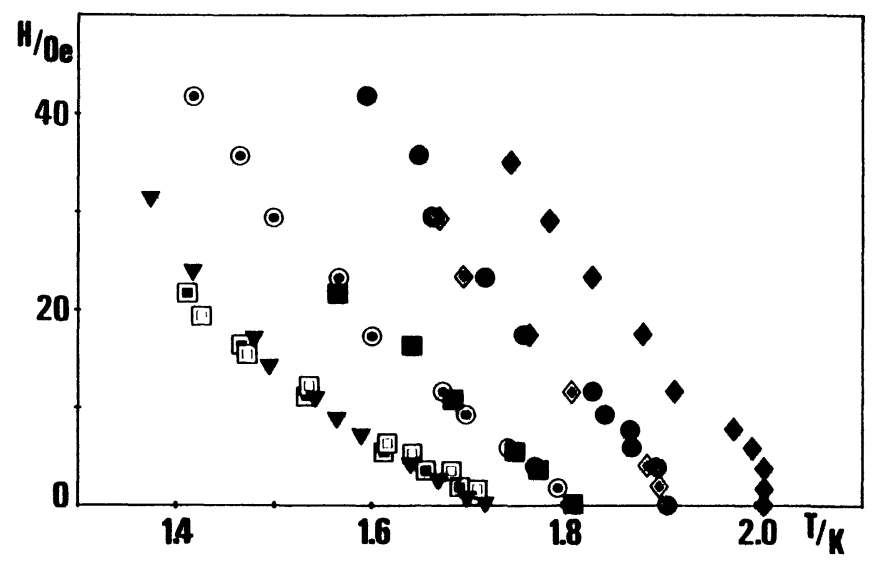

Fig. 2. - H-T lines obtained from $\chi^{\prime \prime}(T, H, \omega)$ data (transverse $\chi_{T}^{\prime \prime}$ and longitudinal $\left.\chi_{L}^{\prime \prime}\right)$ by using the two different $L_{1}$ and $L_{2}$ threshold values for $\chi^{\prime \prime}$. Full symbols refer to $\chi_{T}^{\prime \prime}$ and $L_{1}: 10^{5} \mathrm{~Hz} ; 10^{4} \mathrm{~Hz} ; 10^{3} \mathrm{~Hz}$; $\nabla 10^{2} \mathrm{~Hz}$. Double symbols refer to $\chi_{T}^{\prime \prime}$ and $L_{2}$ (if full) $\diamond 10^{5} \mathrm{~Hz} ; \odot 10^{4} \mathrm{~Hz} ; \square 10^{3} \mathrm{~Hz}$ and to $\chi_{L}^{\prime \prime}$ and $L_{2}$ (if open) 回 $10^{3} \mathrm{~Hz}$.

$T_{\mathrm{G}}\left(0,10^{-1} \omega\right)$. It is perfectly clear on figure 2 that the set of $\left(L_{1}, \omega\right)$ lines coincide with the set of $\left(L_{2}, 10^{-1} \omega\right)$ lines. This means that raising (or lowering) the threshold value $L$ is similar to changing the experimental time scale.

A set of $T_{G}(H)$ lines derived by using a given (low $L_{1}$ ) threshold value for both the longitudinal and transverse configurations are reported on figure 3 in a $\left(g \mu_{\mathrm{B}} H / k T_{\mathrm{G}}^{0}\right)^{2 / 3}$ scale.

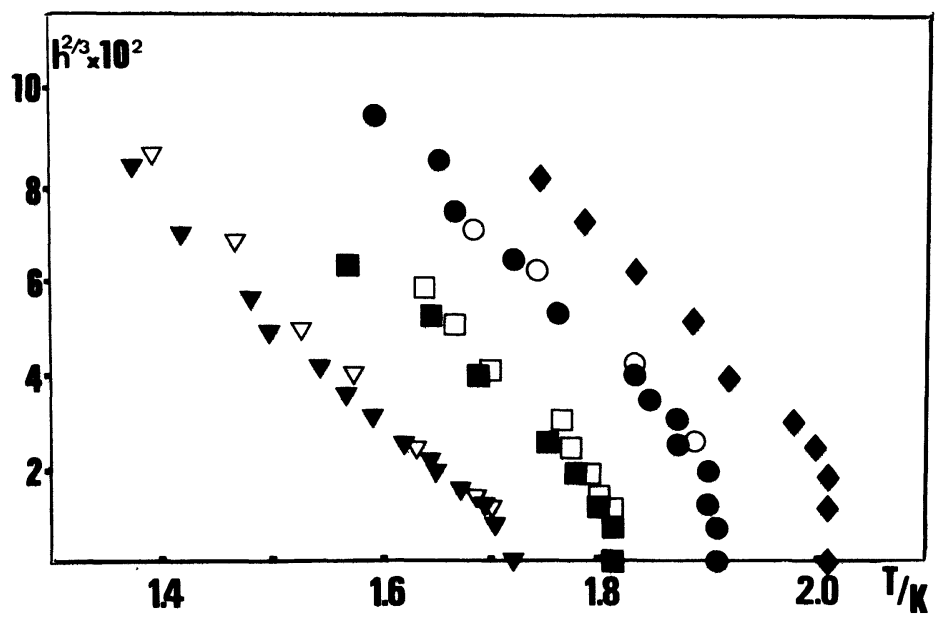

Fig. 3. - Plot of $h-T$ lines on a $h^{2 / 3}$ scale. $h=g \mu_{\mathrm{B}} B H / k T_{\mathrm{G}}^{0}(\omega)$. Full symbols refer to transverse data, open symbols to longitudinal data. $\diamond 10^{5} \mathrm{~Hz} ; O \bullet 10^{4} \mathrm{~Hz} ; \square \square 10^{3} \mathrm{~Hz} ; \nabla \nabla 10^{2} \mathrm{~Hz}$.

There is a clear gradual change of the shape of the line as the frequency is increased (i.e. the time scale is reduced). At the lowest frequency investigated $\left(10^{2} \mathrm{~Hz}\right)$, the experimental points fall on a straight AT line in a $h^{2 / 3}$ scale except perhaps for $T_{\mathrm{G}}^{0}$, which however remains within the experimental error $\left(2 \times 10^{-2} \mathrm{~K}\right)$. This is fully consistent with our previous $1 \mathrm{~ms}$ measurements [7]. 
As the time scale gets shorter, whereas the high field points remain on straight parallel lines, the low field data deviate from this AT behaviour. The higher the frequency, the larger the field where this deviation occurs. Such a behaviour has never been noted in other spin-glasses, but there are very few short time measurements performed until now, to our knowledge, except $[6,7]$.

A second important characteristic of our data is the similarity of the transverse and longitudinal out of phase response : they both lead to the same line. This means that the amount of irreversibility which is measured is the same whatever-the orientation of $\Delta H$ with respect to $H$.

In the longitudinal configuration, the response is related to the standard remanent effects. In the transverse configuration, measuring, as we do, magnetic after-effects, means that the magnetization is not free to rotate, i.e., that there is some anisotropy energy.

We discuss now the origin of the anisotropy and the physical implications. Two origins may be invoked : one is the crystal field through the spin-orbit coupling, another is the dipole-dipole interaction. EPR measurements [17] performed on very dilute $\mathrm{Eu}^{2+}: \mathrm{SrS}$ samples give an order of magnitude of the crystal field anisotropy :

$$
E_{\mathrm{CF}} \sim 0.03 \mathrm{~K} \text {. }
$$

Dipole-dipole interaction between nearest neighbours leads to :

$$
E_{\mathrm{dd}} \sim 0.4 \mathrm{~K} \text {. }
$$

We can thus neglect $E_{\mathrm{CF}}$ compared to $E_{\mathrm{dd}}$.

Dipole-dipole coupling, whose symmetry appears to be different from the DzyaloshinskiiMoriya (DM) anisotropic interactions prevailing in diluted metallic spin-glasses [18] exhibit however some similar features : first there must not be any preferred axis for such an anisotropy ; second, it does only exist if « frozen correlations " are established between the spins. The detailed expression of this energy :

$$
E_{\mathrm{dd}}=\sum_{i j}\left(g^{2} \mu_{\mathrm{B}}^{2} / R_{i j}^{3}\right)\left[\mathbf{S}_{i} \cdot \mathbf{S}_{j}-3\left(\mathbf{S}_{i} \cdot \mathbf{R}_{i j}\right)\left(\mathbf{S}_{j} \cdot \mathbf{R}_{i j}\right) / R_{i j}^{2}\right]
$$

(where $R_{i j}$ specifies the distance between the sites $i$ and $j$ ) implies a coupling between all spin components which is not small $(0.4 \mathrm{~K})$ compared to the characteristic exchange value $\left(T_{\mathrm{G}} \sim\right.$ $1.6 \mathrm{~K}$ ). Therefore, by probing the total response of the system (which is the case in our measurement) we cannot discriminate between the transverse and the longitudinal local irreversible response : the total response must be isotropic; this is indeed what we have observed.

Therefore our results do no necessarily invalidate the predictions of mean field theory, in which the local responses are expected to be different. We might in fact argue that even if any change in $\Delta M$ is isotropic, it must also exhibit the total amount of irreversibility which is present in the system ; in the high temperature region, the irreversibility is assumed to be predominantly transverse [9]. Our experiment does not tell us whether the irreversibility is transverse or not; it just shows that in some way the system is irreversible, up to a line which is rather suggestive of the "Gabay-Toulouse » (GT) line [8]. However the field scale found at $10^{5} \mathrm{~Hz}$ for the line is very small :

$$
h_{\mathrm{exp}} / h_{\mathrm{GT}} \sim 10^{-3}
$$

Moreover, there is presently no mean field calculations including random anisotropies such as the dipolar interactions.

On the other hand, our results are surprisingly similar to the ones obtained in numerical simulations (NS) $[13,14]$ where "AT like " lines have been found for 2D Ising spin systems. In particular, in reference 13, figure 4 shows curves associated to a constant characteristic relaxation time $\tau$. When this time $\tau$ is decreased, the shape of the line changes quite similarly to our data. 
As pointed out to us independently by G. Toulouse and A. P. Young [19], this low-field change of curvature may suggest that the analyticity $\left(T_{\mathrm{G}}(H)-T_{\mathrm{G}}(0) \sim H^{2}\right)$ which exists in the high temperature regime, i.e., at short times for our system, breaks down as the temperature is lowered, as best displayed by figure 2 .

Though the behaviour of our $\mathrm{Eu}_{0.4} \mathrm{Sr}_{0.6} \mathrm{~S}$ is remarkably similar to the 2D system, the field scale is still not satisfactory $: h_{\exp } / h_{\mathrm{Ns}} \sim 3 \times 10^{-2}$.

However, these estimates $\left(h_{\text {exp }} / h_{\mathrm{GT}}\right.$ or $\left.h_{\text {exp }} / h_{\mathrm{NS}}\right)$ must be considered with care : the $\mathrm{Eu}_{0.4} \mathrm{Sr}_{0.6} \mathrm{~S}$ system is highly concentrated; a non-zero ferromagnetic interaction $J_{0}$ is to be expected and it may be large. Unfortunately the high temperature measurements of the paramagnetic Curie temperature $\theta$ gives $\theta \sim 7 \mathrm{~K}$ [20] which is not reasonable since the system becomes a spin-glass at a temperature smaller than $2 \mathrm{~K}$. We are thus left with an experimental field $H /\left(1-J_{0}\right)$ to be compared to the theoretical one (which is larger than the applied one) but not known.

In summary, we do not believe that we can really discriminate between two extreme situations as described by mean field theory or numerical simulations on the sole basis of the shape of our $T_{\mathrm{G}}(H)$ lines and the field scale. There appears to be some reasons to believe that mean field theory breaks down for a dimensionality $d<4$ in short range systems [11, 12]. However, none of the authors have, until now, taken dipolar interactions into account.

We have definitely shown that a reversed concavity appears at low fields when the experimental time scale gets shorter. It is suggested that this dynamical measurement might reveal breakdown of analyticity in the field dependence of the transition temperature as the temperature is lowered. The similarity of the transverse and the longitudinal irreversible response is consistent with an anisotropy energy of the dipole-dipole type. The magnitude of such an energy is not negligible compared to the characteristic exchange energy. This, added to the fact that the symmetry of the dipolar interaction is not exactly the same as for the DM interaction, might be an important difference between metallic and insulating (more concentrated) spin-glass.

\section{Acknowledgments.}

We are most grateful to H. Alloul, R. Orbach, H. Sompolinsky, G. Toulouse and A. P. Young for illuminating remarks. We acknowledge valuable discussions with $\mathrm{P}$. Monod and $\mathrm{H}$. Bouchiat. We like to thank $\mathrm{H}$. Bouchiat for having made possible the determination of demagnetizing factors and $\mathrm{H}$. Maletta for kindly providing us with the sample. We were quite recently informed of a similar work by J. Hamida, C. Paulsen, S. J. Williamson, H. Maletta (private communication). We acknowledge valuable help of A. Noel and Y. Rançon in the early stage of this work.

\section{References}

[1] De Almeida, J. R. L. and Thouless, D. J., J. Phys. A 11 (1978) 983.

[2] Monod, P. and Bouchiat, H., J. Physique Lett. 43 (1982) L-45.

[3] Chamberlin, R. V., Hardiman, M., Turkevich, L. A. and Orbach, R., Phys. Rev. B 25 (1982) 6720.

[4] Berton, A., Chaussy, J., Odin, J., Rammal, R. and Tournier, R., J. Physique Lett. 43 (1982) L-153.

[5] Yeshurun, Y., Ketelsen, L. J. P. and Salamon, M. B., Phys. Rev. B 26 (1982) 1491.

[6] Tholence, J. L. and Salamon, M. B., J. Magn. Magn. Mat. 31-34 (1983).

[7] Bontemps, N., Rajchenbach, J. and Orbach, R., J. Physique Lett. 44 (1983) L-47.

[8] Toulouse, G. and Gabay, M., J. Physique Lett. 42 (1983) L-103.

[9] Cragg, D. M., Sherrington, D. and Gabay, M., Phys. Rev. Lett. 49 (1982) 158.

[10] This point was brought to our attention by H. SOMPOLINSKY.

[11] Sompolinsky, H. and Zippelius, A., Phys. Rev. Lett. 50 (1983) 1297.

[12] Bray, A J. and Moore, M. A., J. Phys. C 12 (1979) 79. 
[13] Young, A. P., Phys. Rev. Lett. 50 (1983) 917.

[14] KinZel, W. and Binder, K., Phys. Rev. Lett. 50 (1983) 1509.

[15] Ferre, J., Rajchenbach, J. and Maletta, H., J. Appl. Phys. 52 (3) (1981) 1697.

[16] Huser, D., Wenger, E., van Duynevelt, A. J. and Mydosh, J. A., Phys. Rev. B 27 (1983) 3100.

[17] Low, W., Phys. Rev. 98 (1955) 426.

[18] FerT, A. and Levy, P.M., Phys. Rev. Lett. 44 (1980) 1538.

[19] Young, A. P., Toulouse, G., (Private communications).

[20] KöBleR, U. and Binder, K., J. Magn. Magn. Mat. 15-18 (1980) 313. 